\title{
Comparison of closed loop and sensorless adaptive optics in widefield optical microscopy
}

C. Bourgenot

C. D. Saunter

G. D. Love

J. M. Girkin

j.m.girkin@durham.ac.uk
Department of Physics, Durham University, South Road, Durham, DH1 3LE, UK

Department of Physics, Durham University, South Road, Durham, DH1 3LE, UK

Department of Physics, Durham University, South Road, Durham, DH1 3LE, UK Biophysical Sciences Institute, Durham University, South Road, DH1 3LE, UK

Department of Physics, Durham University, South Road, Durham, DH1 3LE, UK Biophysical Sciences Institute, Durham University, South Road, DH1 3LE, UK

We report on a closed loop widefield adaptive optics, optical microscopy system in which the feedback signal is provided by backscattered light from the sample acting as a guide star. The improvement in imaging performance is compared to an adaptive optics system controlled via an image optimisation routine commonly described as sensorless adaptive optics. The samples viewed were imaged without fluorescence to ensure that photobleaching and other potential variations did not affect the comparisons in system performance though the method is equally applicable for fluorescence microscopy. The closed loop system is self-optimising for different areas of the sample, using a common reference wavefront, with the accuracy of the loop being limited by variation across the sub-aperture images induced by guide star elongation. Optimisation using an image sharpness metric gives slightly sharper images but takes significantly longer. We thus believe that both wavefront sensor based closed loop AO and metric based optimisation have a role to play in AO for microscopy and that the method of backscattered light as a guide star has a great potential in the application of A0, particularly to optical coherence tomography. [DOI: http://dx.doi.org/10.2971/jeos.2013.13027]

Keywords: Optical microscopy, adaptive optics, widefield microscopy, sensorless adaptive optics, closed loop adaptive optics

\section{INTRODUCTION}

Optical microscopy and astronomy have always played a leading role in the development of optical systems and this tradition continues to this day. In both fields the ultimate diffraction limit of an imaging system is rarely reached as aberrations in the optical system perturb the image. Generally in modern systems these aberrations are neither due to the optical components nor their alignment, being instead induced by the medium through which the instrument has to operate. In the case of astronomy this is the atmosphere, and in the case of optical microscopy the sample through which you are imaging. In both classes of instrument the optical paths contain changes in the local refractive index of the material, which affect the light path and wavefront of the light reaching the detector leading to a loss of spatial resolution and contrast in the image. In microscopy these wavefront aberrations become more significant with depth into the sample, limiting high resolution imaging to shallow depths. Over the last ten years there has been significant growth in the development and application of adaptive optics methods within optical microscopy to overcome these limitations on the imaging depth and resolution in biological samples. This interest has partly been fuelled by the development of novel optical microscopy methods, but also in the significant reduction in cost of adaptive optical elements such that they are no longer only within reach of highly funded optical telescopes. The challenge of improved imaging in microscopy, although having clear similarities with astronomical observations, also has a number of significant differences. Crucially in the astronomical case the aberrations are rapidly time varying whereas in most microscopy cases, even for in vivo imaging, the sample variations change less rapidly in time. Thus in the microscopy field the main route being followed uses an optimisation methodology based upon an image metric rather than actually sampling the aberrations within the sample in real time, as is generally done in astronomy.

The original work on the application of adaptive optics to optical microscopy was focused upon beam scanned optically sectioned microscopy both in confocal and non-linear modalities [1]-[4] and the application in both structured illumination [5] and conventional widefield microscopy [6] or fluorescent lightsheet microscopy [7] followed the initial work. All of these results used some form of image optimisation based algorithm in which an image metric (sharpness, brightness, contrast being typical examples) was maximised by running through a range of mirror shapes. Whilst this method clearly worked in the temporally slowly varying microscopic image, a method using a measurement of the actual wavefront aberration, and hence correction via a closed loop system, was first shown by [8] using a back scattered source as the guide star and subsequently a fluorescent beacon [9] and [10]. A complete review of the work undertaken is well beyond the scope of this paper but the full range of methods and variations employed can be found in [11] where the background to image aberrations in optical microscopes is well explained along with an outline of the techniques employed and [12], which 
covers the practical implementation in particular in complex biological samples.

We report on a closed loop-AO system that adapts methods developed for ophthalmic AO in a way that is applicable to a number of optical microscopy systems encompassing both scanned and widefield (camera based) modalities ${ }^{1}$. The novelty of this work compared to previous publications (on sensored configurations using backscattered light) is, in particular:

- The use of an independent laser probe which can be positioned anywhere in the field of view (assuming the observed region of the sample presents enough backscattered light).

- The use of image optimisation to define the null position for the wavefront sensor, which allows a calibration in situ, without the need to remove the sample.

Furthermore, an important feature of our design is that it overcomes the so called double pass effect which occurs when the guide star beam propagates through the aberrating media to its focus, where it is reflected before passing a second time, in the reverse direction, through the same aberrating media. The result of this double pass is that the odd coefficient aberrations varying with aperture such as coma, trefoil etc. are cancelled and the WFS is thus therefore unable to sense them. This double path effect has been widely analysed for ophthalmic AO $[13,14]$. One solution, which we utilise in this paper, is to use a small diameter laser probe, which only passes through a small part of the pupil, but after scattering the returned light passes through the full pupil [15]. In addition, the incoming beam is also subjected to direct reflection from intermediate surfaces such as the cornea in ophthalmic AO, or the glass cover slip in microscopy. These reflections generate extra sets of intense spots on the WFS camera. In the case of ophthalmic $\mathrm{AO}$ a solution is to use an off-axis beam. Here, we demonstrate a method using the change in light polarisation during the scattering process to discriminate the light coming from specular (direct reflection) and diffuse reflection (elastic scattering).

The advantage of using backscattered light as the reference wavefront from within the sample, compared to fluorescence for example, is that one is not limited by the presence of an inserted fluorescent beacon which, a) is potentially perturbing the sample, b) may not be in the correct position for the structure that one wishes to view, c) may suffer from photobleaching effects. In our system a single narrow laser beam is directed into the back aperture of the microscope objective onto the area of interest within the sample and the back-scattered light is directed onto a WFS after being reflected off the AO element to ensure a closed loop configuration. The performance of the closed loop system was then compared to the more widely used sensorless approach in optical microscopy. The experimental system did not operate in fluorescence as we wished to ensure that the comparative measurements made using the different $\mathrm{AO}$ modalities were not influenced by fluorophore degradation, which can be a complication even in

\footnotetext{
${ }^{1}$ It should be noted that we use the term widefield as defined in the microscopy community, meaning a non-beam scanned technique.
}

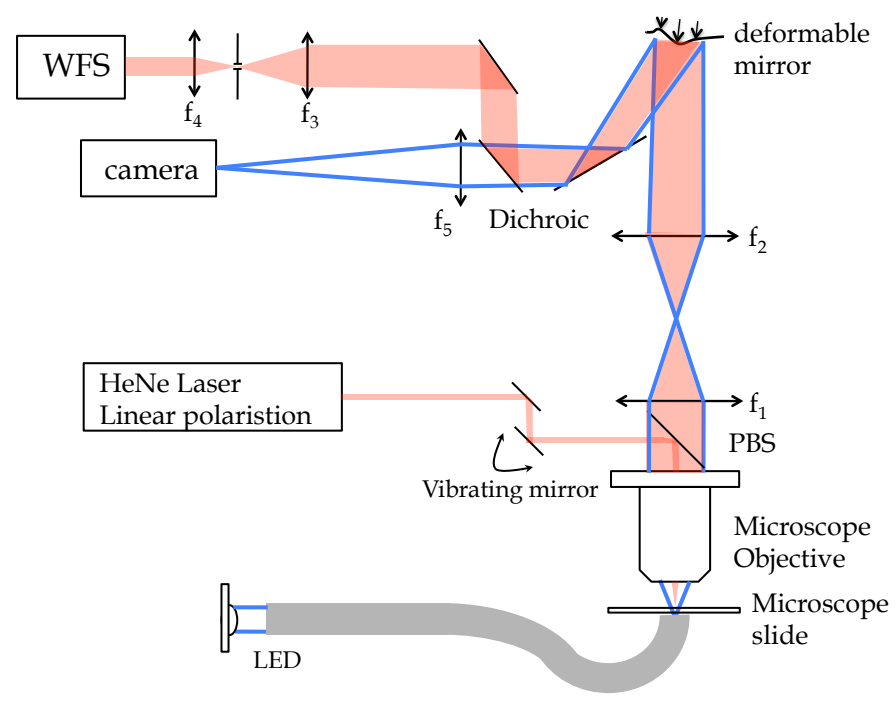

FIG. 1 Optical configuration of the closed loop adaptive optics microscope.

widefield optical microscopy. The system, however, is easily converted to more conventional biological microscopy configurations.

\section{EXPERIMENTAL METHOD AND DETAILS}

The basic optical configuration is shown in Figure 1. The sample illumination was provided by a blue LED (wavelength centred at $470 \mathrm{~nm}$ ) via a liquid light guide to ensure maximum étendue and even illumination of the sample. Imaging light was collected by a Nikon Fluor (x100, NA 1.30) objective. However, the illumination NA was less than the microscope objective collection NA and was estimated to be 0.84 . Epi-illumination was not used due to the slight complication of the laser probe coupling but could be incorporated in future designs.

The localised guide star was produced using the output from a sub $\mathrm{mW} 633 \mathrm{~nm}$ linearly polarised HeNe laser with a beam diameter of $1.5 \mathrm{~mm}$. This is relatively small compared to the $5.2 \mathrm{~mm}$ back aperture of the microscope objective used. The light from the laser was reflected from a vibrating mirror to remove the speckle that otherwise appeared on the wavefront sensing camera [16]. By oscillating the mirror over a very small angle at $120 \mathrm{~Hz}$, compared to the $10 \mathrm{~Hz}$ frame rate of the sensing camera, the speckle is effectively removed by temporal averaging. After passing off the vibrating mirror the laser light was directed into the back aperture of the objective using a polarised beam splitter (PBS) to be focused down to the position of interest on the sample.

Scattered laser light from the sample was then collected using the same objective lens and the back aperture of the objective was re-imaged through a telecentric configuration composed of 2 lenses $\left(f_{1}=200 \mathrm{~mm}\right.$ and $\left.f_{2}=400 \mathrm{~mm}\right)$ onto the deformable mirror (DM), an Imagine Optics Mirao 52-e. After the DM, a dichroic beam-splitter sent the backscattered laser light onto the Shack-Hartman WFS (Thorlabs WFS150-7AR) via two lenses $\left(f_{3}=400 \mathrm{~mm}\right.$ and $\left.f_{4}=75 \mathrm{~mm}\right)$ again working in a telecentric arrangement. The pupil of the system was 
sampled with 97 sub-apertures. The WFS sub-aperture dimension and focal length were respectively $0.15 \mathrm{~mm}$ and $5 \mathrm{~mm}$. A $850 \mu \mathrm{m}$ diameter pinhole was placed confocally between $f_{3}$ and $f_{4}$ to filter light scattered by tissue located away from the focal plan of the microscope objective, with the pinhole being sized to afford the maximum possible depth discrimination without spatially filtering the wavefront. The depth resolution at the imaging plane induced by this pinhole was $7 \mu \mathrm{m}$. Finally, the imaging beam was transmitted by the dichroic beamsplitter and focused by a lens $\left(f_{5}=200 \mathrm{~mm}\right)$ for detection on the science camera (Q-imaging Retiga 1300).

As our system does not employ optical sectioning a relatively thin sample was used compared to previous examples of $\mathrm{AO}$ in microscopy to prevent out of focus layers from obscuring the effect of AO correction. The image could be made optically sectioned through the use of structured illumination but this was not attempted in this work, as the aim was to demonstrate the effectiveness of a backscattered guide star and compare the performance with an optimisation approach. The sample was a 4-5 microns thick mouse back skin tissue (C57bl6/CBA F1 mice) stained with Hematoxylin and Eosin.

\section{CALIBRATION AND CONTROL}

A reference beam from a separate source can be used to provide a null reference position for the WFS spots but this is prone to non-common path errors [17]. Instead we used a wavefront sensorless configuration to define the null (or optimal) wavefront and then compared WFS and sensorless op-

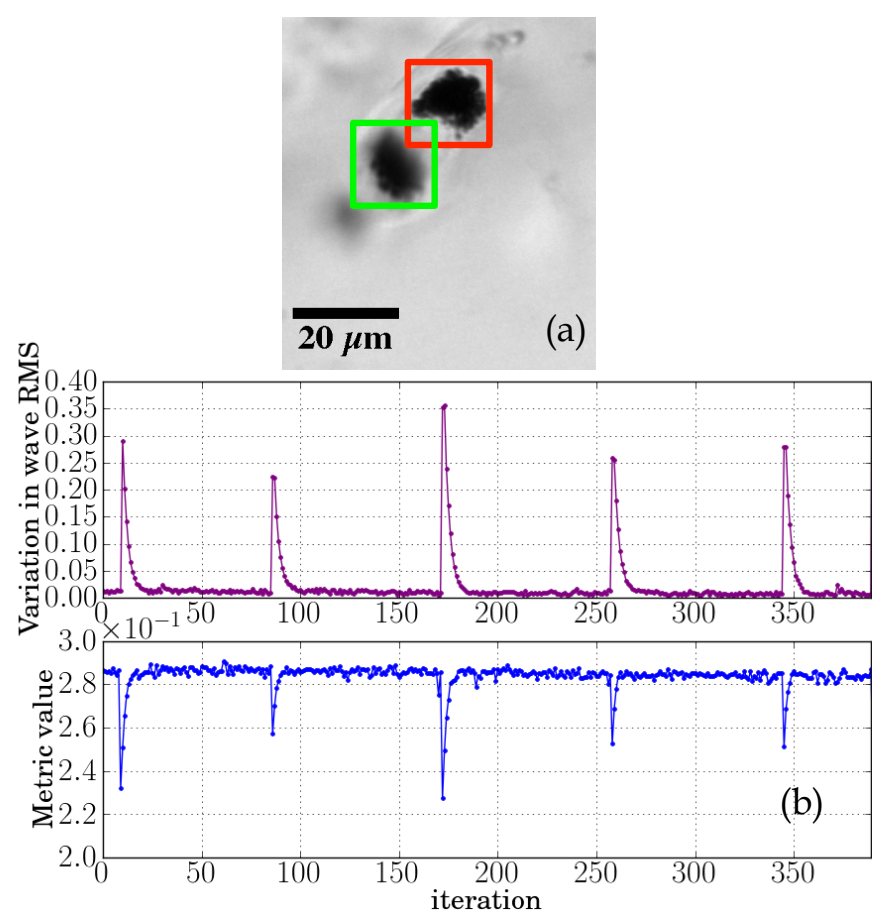

FIG. 2 (a) Image of melanin dots in a hair follicle in back mouse tissue, on which the optimisation and closed loop are run. The red square corresponds to sample 1 and the green square to sample 2. The reference wavefront is recorded on the optimised image of sample 1. (b) Recorded variation on the RMS wavefront (top) and metric value while the closed-loop is on for the sample 1 , and when 5 sets of random perturbations are sent to the mirror. eration. The DM was controlled using a simplex optimisation algorithm with an edge detection maximisation (based on a Sobel filter) as the metric and once the sample was in place, the optimisation was run, enabling the correction of 0.3 wave RMS of aberration. We then recorded a reference wavefront, from the backscattered HeNe light, to define the reference wavefront for the closed loop system. The initial imaging area, for example sometimes being a black melanin spot in a mouse back skin hair follicle, is shown in the red box in Figure 2(a) (later referred to as sample 1).

With the wavefront, corresponding to this optimal image, the AO loop was then closed with the WFS now determining the correction to be placed on the mirror. The stability of the loop was then checked by sending random aberrations composed of a set of low order Zernike modes to the DM to perturb the system with focus being intentionally added to the random aberration. For each step, the RMS wavefront was recorded as well as the metric value. Once the stability of the loop had been confirmed, we then paused the control loop whilst the sample was translated to place another melanin spot (later referred to as sample 2) in the region of interest coincident with the laser guide star (green box on Figure 2(a)). The closed loop was then re-activated on sample 2 , still using the reference wavefront recorded from sample 1. After recording the response of the closed loop system, the experiment was repeated only using an image based optimisation routine to determine the best mirror shape.

\section{RESULTS}

The initial calibration images and loop stability data are shown in Figure 2. It should be noted on Figure 2(a) that, while sample 1 is in focus, sample 2 is seen blurred because it is not at the same depth as sample 1. The top section of Figure 2(b) shows the variation of the RMS wavefront for 5 random perturbations when the closed loop control is enabled and the bottom chart shows the metric variation during the loop. For each perturbation, the system restores the minimum wavefront error and the maximum metric value within around 5 iterations. It has to be noted that no effort has been placed in optimising the speed of the closed loop AO system which is currently limited to $5 \mathrm{~Hz}$ by the software interface to the WFS. However, the fact that the returning scattered light intensity can be adjusted with the laser probe intensity without impacting the imaging path allows for much higher closed loop rates with a suitable WFS camera, presenting a major advantage over fluorescent beacons, which are usually limited by the number of fluorescence photons received on the WFS camera.

The second sample area was then placed into the system as described above. No manual refocusing from sample 1 to 2 was performed, and this correction was left to the AO system. Figure 3(a) shows the wavefront residual error in wave RMS (top chart), from the null position recorded after image optimisation on sample 1, and the image sharpness value (bottom chart) quickly converging to their optimal values. The metric value is the favoured criteria for the representation of image quality because, due to the initial poor quality of the 

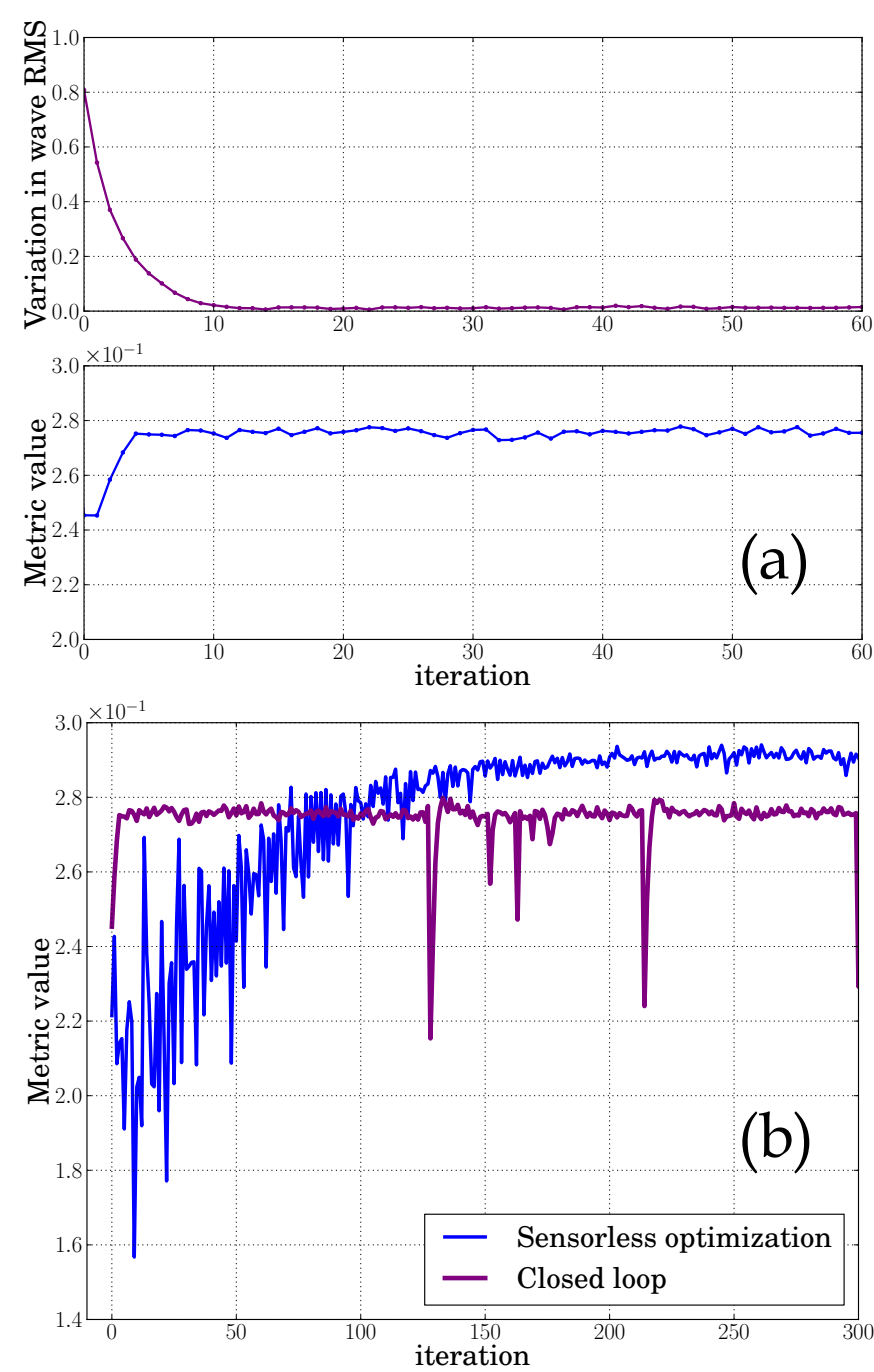

FIG. 3 In (a), RMS wavefront (top) and metric value (bottom) when the closed loop is enabled on sample 2 using the reference wavefront recorded on sample 1. On (b), metric value comparison while the system is optimising on sample 2 in a sensorless configuration using the simplex (in blue), and in a WFS configuration (in violet). After reaching the plateau (at iteration 5), in the closed loop configuration, the stability of the system is checked by sending random aberration onto the mirror, leading to downwards spikes in metric value after iteration 100.

wavefront sensor spots, the indirect estimation of the phase aberration with the wavefront sensor inherently has a large associated error (which is not estimated here). Furthermore, the asymptotical closed loop convergence value is not rigorously zero (0.015 wave RMS), leading to further source of error. As the melanin spot used for sample 2 is different from the one used for the calibration and the stability and repeatability tests above, the absolute metric value between Figures 2(b) and 3(a) cannot be directly compared. Thus we have demonstrated that we can move the sample and subsequently observe, any arbitrary part of the object removing the resulting aberrations using the original wavefront measurement as the target for the closed loop. In Figure 3(b) the AO corrected images of sample 2 with the closed loop and with the image sharpness metric performed are compared. The closed loop optimisation is very fast, typically reaching the plateau in 5 iterations. The sensorless optimisation does lead to a slightly higher metric value, and thus a sharper image, but this takes on average 250 iterations compared to 3 iterations for the sen-

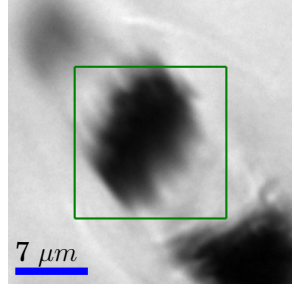

(a)

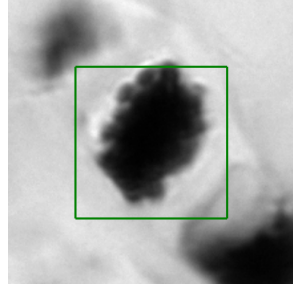

(b)

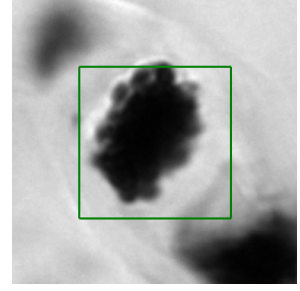

(c)
FIG. 4 Image of sample 2 before optimisation (a), when the closed loop is ON (b) and after a sensorless optimisation (c). The square delimits the widefield optimisation area during sensorless optimisation.

sored configuration. However, it has to be noted that the convergence speed for the sensorless approach depends also on the algorithm used for correction [18]. Here we used a NedlerMead Simplex algorithm, and the iteration count could be reduced with a modal optimisation algorithm [7]. It is notable that any sensorless approach needs a great many more iterations than the WFS approach.

In Figure 3(b), once the closed loop system has reached the plateau, random aberrations are sent to the mirror to check the stability. Each time, the closed loop restores the optimal metric value within 5 iterations. Images on Figure 4 are for sample 2 when the DM is flat (a), and optimised using closed loop correction (b) and sensorless optimisation (c). Although, the sensorless and WFS configurations lead to very similar images, the image after optimisation with the sensorless configuration is slightly better than the one obtained with the closed loop. This result is also confirmed by the image sharpness metric value being at a higher level in the case of the sensorless optimisation. We hypothesise that the lower metric value is the result of three effects:

- Partial sampling of the pupil by the WFS sub-apertures leads to a partial correction of the wavefront distortion. For a given pupil diameter, there are some areas, at the edge of the pupil, which are not covered by a subaperture.

- Field variant WFS images. The laser guide star illuminates a 3 dimensional volume within the sample, which is then viewed by each WFS microlens from a different angle and through a different path, distorting WFS images in a similar manner to LGS elongation in astronomical AO $[19,20]$. The problem may be exacerbated in microscopy by inhomogeneous absorption through the intervening tissue.

- Given that the sensorless configuration was allowed to optimise a range of modes including defocus, the axial position of the image could also vary, whilst for the sensored case, the axial position was fixed by the confocal pinhole in the sensing arm. As a result, the search space was larger for the image optimisation, leading to a slightly higher metric. 


\section{CONCLUSION}

We have demonstrated the use of partial confocal back scattered light from the sample as a reference laser guide star source for a WFS in a closed loop AO microscope. We have implemented an optical configuration that removes the double path effect as well as the effects of spurious guide star reflections, such as those from the slide and lens surface, which would otherwise confuse the WFS by adding extra sets of spots. We have demonstrated the initial use of an optimisation technique to provide a reference wavefront for much faster WFS based AO. The system has then been shown to operate in a true closed loop manner as we move around the sample with the WFS ensuring an optimal image. The accuracy of the loop is limited by the contribution of the spot shape to the centre of mass measurement and also by the partial covering of the pupil by the sub-apertures leaving the very edge of the pupil uncorrected. The speed has not been optimised here, but would be significantly improved with a faster WFS camera. Optimisation using image sharpness metric gives slightly better results but takes significantly longer (requiring about 50 times more iterations and therefore images). We thus believe that both true closed loop $\mathrm{AO}$ and metric based optimisation have a role to play in $\mathrm{AO}$ in microscopy.

\section{ACKNOWLEDGEMENTS}

The authors wish to acknowledge funding from the British Heart Foundation, in particular a Research Excellence Award, and the Engineering and Physical Science Research Council. All samples were used in accordance with UK Home Office rules. The sample (murine dorsal tissue incorporating GFP expressing skin stem cells) has been supplied by Dr Carrie Ambler and Dr Richie Wong (Durham University).

\section{References}

[1] 0. Albert, L. Sherman, G. Mourou, T. B. Norris, and G. Vdovin, "Smart microscope: an adaptive optics learning system for aberration correction in multiphoton confocal microscopy," Opt. Lett. 25, 52-54 (2000).

[2] M. J. Booth, M. A. A. Neil, R. Juskaitis, and T. Wilson, "Adaptive aberration correction in a confocal microscope," P. Natl. Acad. Sci. USA 99, 5788-5792 (2002).

[3] P. Marsh, D. Burns, and J. Girkin, "Practical implementation of adaptive optics in multiphoton microscopy," Opt. Express 11, 1123-1130 (2003).

[4] A. J. Wright, S. P. Poland, J. M. Girkin, C. W. Freudiger, C. L. Evans, and X. S. Xie, "Adaptive optics for enhanced signal in CARS microscopy," Opt. Express 15, 18209-19 (2007).

[5] D. Débarre, E. J. Botcherby, M. J. Booth, and T. Wilson, "Adaptive optics for structured illumination microscopy," Opt. Express 16, 9290-305 (2008).
[6] B. Potsaid, Y. Bellouard, and J. Wen, "Adaptive Scanning Optical Microscope (ASOM): A multidisciplinary optical microscope design for large field of view and high resolution imaging," Opt. Express 13, 462-467 (2005).

[7] C. Bourgenot, C. D. Saunter, J. M. Taylor, J. M. Girkin, and G. D. Love, "3D adaptive optics in a light sheet microscope," Opt. Express 20, 13252-61 (2012).

[8] J. W. Cha, J. Ballesta, and P. T. C. So, "Shack-Hartmann wavefront-sensor-based adaptive optics system for multiphoton microscopy," J. Biomed. Opt. 15, 046022 (2012).

[9] 0. Azucena, J. Crest, S. Kotadia, W. Sullivan, X. Tao, M. Reinig, D. Gavel, S. Olivier, and J. Kubby, "Adaptive optics wide-field microscopy using direct wavefront sensing," Opt. Lett. 36, 825-827 (2011).

[10] P. Vermeulen, E. Muro, T. Pons, V. Loriette, and A. Fragola, “Adaptive optics for fluorescence wide-field microscopy using spectrally independent guide star and markers," J. Biomed. 0pt. 16, 076019 (2011).

[11] M. J. Booth, "Adaptive optics in microscopy," Philos. T. R. Soc. A $365,2829-43$ (2007).

[12] J. M. Girkin, S. Poland, and A. J. Wright, "Adaptive optics for deeper imaging of biological samples," Curr. Opin. Biotech. 20, 106-110 (2009).

[13] P. Artal, S. Marcos, R. Navarro, and D. Williams, "Odd aberrations and double-pass measurements of retinal image quality," J. Opt. Soc. Am. A 12, 195-201 (1995).

[14] L. Diaz-Santana and J. C. Dainty, "Effects of retinal scattering in the ocular double-pass process," J. Opt. Soc. Am. A 18, 1437-44 (2001).

[15] J. Liang, B. Grimm, S. Goelz, and J. F. Bille, "Objective measurement of wave aberrations of the human eye with the use of a HartmannShack wave-front sensor," J. Opt. Soc. Am. A 11, 1949-1957 (1994).

[16] M. Rueckel, J. A. Mack-Bucher, and W. Denk, “Adaptive wavefront correction in two-photon microscopy using coherence-gated wavefront sensing," P. Natl. Acad. Sci. USA 103, 17137-42 (2006).

[17] N. P. Doble, G. D. Love, D. F. Buscher, R. M. Myers, and A. Purvis, "Use of image quality metrics for correction of noncommon path errors in the ELECTRA adaptive optics system," Proc. SPIE 3749, 785-786 (1999).

[18] A. J. Wright, D. Burns, B. A. Patterson, S. P. Poland, G. J. Valentine, and J. M. Girkin, "Exploration of the optimisation algorithms used in the implementation of adaptive optics in confocal and multiphoton microscopy," Microsc. Res. Techniq. 67, 36-44 (2005).

[19] K. J. Jones, "NA variability and LGS elongation: impact on wavefront error," Proc. SPIE 8149, 81490E-81490E-9 (2011).

[20] C. Robert, J.-M. Conan, D. Gratadour, C. Petit, and T. Fusco, "ShackHartmann tomographic wavefront reconstruction using LCS: analysis of spot elongation and fratricide effect," 1St A04ELT conference - Adaptive Optics for Extremely Large Telescopes 05010, 05010 (2010). 